\title{
THE DEPTH OF CENTRES OF MAPS ON DENDRITES
}

\author{
HISAO KATO \\ (Received 10 October 1995; revised 25 March 1997)
}

Communicated by J. A. Hillman

\begin{abstract}
Xiong proved that if $f: I \rightarrow I$ is any map of the unit interval $I$, then the depth of the centre of $f$ is at most 2, and Ye proved that for any map $f: T \rightarrow T$ of a finite tree $T$, the depth of the centre of $f$ is at most 3. It is natural to ask whether the result can be generalized to maps of dendrites. In this note, we show that there is a dendrite $D$ such that for any countable ordinal number $\lambda$ there is a map $f: D \rightarrow D$ such that the depth of centre of $f$ is $\lambda$. As a corollary, we show that for any countable ordinal number $\lambda$ there is a map (respectively a homeomorphism) $f$ of a 2-dimensional ball $B^{2}$ (respectively a 3-dimensional ball $B^{3}$ ) such that the depth of centre of $f$ is $\lambda$.
\end{abstract}

1991 Mathematics subject classification (Amer. Math. Soc.): primary 54H20; secondary 58F50. Keywords and phrases: Dendrite, non-wandering points, centre, depth.

\section{Introduction}

In [6], Xiong proved that if $f: I \rightarrow I$ is any map of the unit interval $I=[0,1]$, then the depth $d(f)$ of the centre of $f$ is at most 2, and in [7], Ye proved that for any map $f: T \rightarrow T$ of a (finite) tree $T$, the depth $d(f)$ of centre of $f$ is at most 3. It is natural to ask whether the result can be generalized to maps of dendrites. In [5], Neumann proved that for any $C^{\infty} n$-manifold $M$ with $n \geq 3$ and any countable ordinal number $\lambda$, there is a $C^{\infty}$ flow $\phi$ on $M$ such that the depth of centre of the flow $\phi$ is $\lambda$.

In this note, firstly we study the depth of centre of maps of 0-dimensional compacta. As corollaries, we show the following:

(1) There is a dendrite $D$ such that for any countable ordinal number $\lambda$ there is a map $f: D \rightarrow D$ such that the depth $d(f)$ of centre of $f$ is $\lambda$.

(2) For any countable ordinal number $\lambda$ there is a map $f$ of a disk $B^{2}$ such that $d(f)=\lambda$.

(C) 1998 Australian Mathematical Society $0263-6115 / 98 \$ A 2.00+0.00$ 
(3) For any countable ordinal number $\lambda$ there is a homeomorphism $h: B^{3} \rightarrow B^{3}$ of a 3-dimensional ball $B^{3}$ such that $h \mid \partial B^{3}=\mathrm{id}, d(h)=\lambda$ and $\Omega_{\lambda}(h)=\partial B^{3} \bigcup Z$, where $Z$ is a compact countable set in $B^{3}-\partial B^{3}$.

All spaces considered in this note are assumed to be separable metric spaces. By a continuum, we mean a non-empty, compact, connected, metric space. Let $I$ be the unit interval $[0,1]$. A tree is a 1-dimensional connected compact polyhedron which contains no simple closed curve. A continuum $D$ is a dendrite if $D$ is a locally connected continuum and $D$ contains no simple closed curve (see [4] for topological properties of dendrites). A point $e$ of a dendrite $D$ is called an end point if there is no subset $A$ of $D$ such that $e \in A$ and $A$ is homeomorphic to the open interval $(0,1)$. Let $E(D)$ be the set of all end points of $D$. Note that a compactum $X$ is a dendrite if and only if $X$ is a 1-dimensional compact absolute retract (=AR). The dynamics of maps (=continuous functions) of $I$ and trees are considerably well-understood. Recently, the dynamical behavior of maps of dendrites have often appeared in Julia sets of complex dynamical systems.

Let $X$ be a compact metric space with metric $d$ and $f: X \rightarrow X$ a map. A point $x \in X$ is a periodic point of $f$ if there is a natural number $n \geq 1$ such that $f^{n}(x)=x$. A point $x \in X$ is a recurrent point of $f$ if for each $\epsilon>0$ there is a natural number $n \geq 1$ such that $d\left(f^{\prime \prime}(x), x\right)<\epsilon$. A point $x \in X$ is a non-wandering point of $f$ if for any neighborhood $U$ of $x$ in $X$ there is a natural number $n \geq 1$ such that $f^{\prime \prime}(U) \cap U \neq \emptyset$. By $P(f)$, we mean the set of all periodic points of $f$, and by $R(f)$ the set of all recurrent points of $f$. Also, the set of non-wandering points of $f$ will be denoted by $\Omega(f)$. The notions of periodic points, recurrent points and non-wandering points are very important in the study of dynamical systems. Note that $P(f) \subset R(f) \subset \Omega(f), \Omega(f)$ is a closed subset of $X$ and $f(\Omega(f)) \subset \Omega(f)$.

Let $\Omega_{0}(f)=X$ and $\Omega_{1}(f)=\Omega(f)$. For any ordinal number $\lambda \geq 1$, recursively we will define $\Omega_{\lambda}(f)$ as follows: If $\lambda=\alpha+1$, then we set $\Omega_{\lambda}(f)=\Omega\left(f \mid \Omega_{\alpha}(f)\right)$. If $\lambda$ is a limit ordinal number, we set $\Omega_{\lambda}(f)=\bigcap_{\alpha<\lambda} \Omega_{\alpha}(f)$.

Then we see that there is a countable ordinal number $\gamma$ such that $\Omega_{\gamma}(f)=$ $\Omega_{\gamma+1}(f)(=\overline{R(f)})$. The minimal such $\gamma$ is called the depth of the centre of $f$, and it is denoted by $d(f)$. Note that $d(\mathrm{id})=0$. In general, it is difficult to determine the centre $\Omega_{\gamma}(f)(=\overline{R(f)})$ and the depth $d(f)$ of the centre of $f$. We are interested in the depth $d(f)$ of the centre of a map $f$.

Let $X$ be a compactum with metric $d$. Then

$$
2^{X}=\{A \mid A \text { is a non-empty closed subset of } X\}
$$

is the hyperspace with the Hausdorff metric $d_{H}$, that is,

$$
d_{H}(A, B)=\inf \{\epsilon>0 \mid A \subset U(B, \epsilon), B \subset U(A, \epsilon)\}
$$


where $U(A, \epsilon)$ is the $\epsilon$-neighborhood of $A$ in $X$. Note that $2^{X}$ is a compact metric space with the metric $d_{H}$.

\section{The depth of centres of maps of compact countable sets}

In this section, we study the 0 -dimensional case. We prove the following.

PROPOSITION 2.1. For any countable ordinal number $\lambda$ there is a compact countable set $Z_{\lambda}$ and a homeomorphism $f_{\lambda}: Z_{\lambda} \rightarrow Z_{\lambda}$ such that $d\left(f_{\lambda}\right)=\lambda$.

PROOF. Note that $d$ (id) $=0$. Recursively, for any countable ordinal number $\lambda>0$ we will construct a compact countable set $Z_{\lambda}$ and a homeomorphism $f_{\lambda}: Z_{\lambda} \rightarrow Z_{\lambda}$ such that $d\left(f_{\lambda}\right)=\lambda$. Let $\omega$ be the first infinite ordinal number and $\mathbb{Z}$ the set of integers. Firstly, we consider the case that $\lambda$ is not a limit ordinal.

I(1): Case of $\lambda=1$. Let

$$
Z_{1}=\left\{x_{1}(n) \mid n \in \mathbb{Z}\right\} \oplus\{x(\infty)\} .
$$

where $x_{1}(i) \neq x_{1}(j)(i \neq j)$ and $\oplus$ implies the disjoint union. Then we can define a metric $d_{1}$ on $Z_{1}$ satisfying $\lim _{n \rightarrow \infty} x_{1}(n)=x(\infty)=\lim _{n \rightarrow \infty} x_{1}(-n)$ (see Figure 1). Define a function $f_{1}: Z_{1} \rightarrow Z_{1}$ by $f_{1}\left(x_{1}(n)\right)=x_{1}(n+1), f_{1}(x(\infty))=x(\infty)$. Note that $Z_{1}$ is compact and $f_{1}$ is continuous. Then $d(f)=1$.

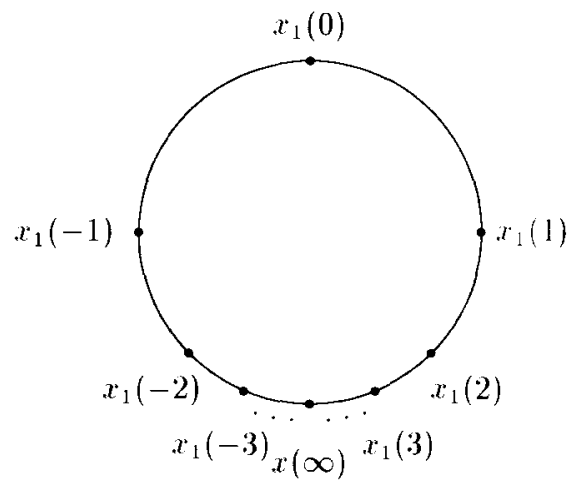

FiGURE 1

$\mathrm{I}(m+1)$ : Case of $\lambda=m+1(1 \leq m<\omega)$. We assume that the set $Z_{m}$, a metric $d_{m}$ on $Z_{m}$ and a homeomorphism $f_{m}$ of $Z_{m}$ have been obtained. Let

$$
Z_{m+1}=Z_{m} \oplus\left\{x_{m+1}(n) \mid n \in \mathbb{Z}\right\},
$$


where $x_{m+1}(i) \neq x_{m+1}(j)(i \neq j)$. Define a function $f_{m+1}: Z_{m+1} \rightarrow Z_{m+1}$ by $f_{m+1}\left(x_{m+1}(n)\right)=x_{m+1}(n+1), f_{m+1} \mid Z_{m}=f_{m}$. Then we can define a metric $d_{m+1}$ on $Z_{m+1}$ such that $d_{m+1}$ is an extension of $d_{m}, \lim _{n \rightarrow \infty} x_{m+1}(-n)=x(\infty) \in Z_{1}$,

$$
\lim _{n \rightarrow \infty}\left(d_{m+1}\right)_{H}\left(\mathrm{Cl}\left(\left\{x_{m+1}(j) \mid j \geq n\right\}\right), Z_{m}\right)=0
$$

and $\Omega\left(f_{m+1}\right)=Z_{m}$ (see Figure 2). Hence $d\left(f_{m+1}\right)=m+1$. Note that $Z_{m} \subset Z_{m+i}$ for each $i \geq 0$.

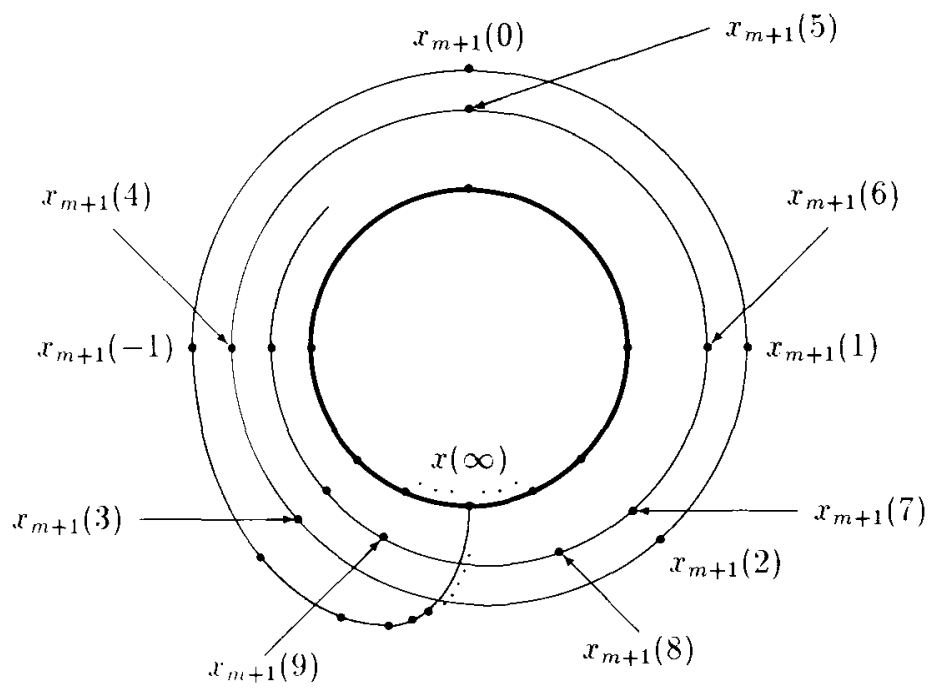

Figure 2

Next, we consider the case that $\lambda$ is a countable ordinal number which is not limit.

$\mathrm{I}(\omega+m)$ : Case of $\lambda=\omega+m$, where $1 \leq m<\omega$. Consider the set $Z_{m}$ and a metric $d_{m}$ on $Z_{m}$ (see the case $\mathrm{I}(\mathrm{m})$ ). Take a sequence $Z_{m+1}, Z_{m+2}, \ldots$ of sets and a sequence $d_{m+1}, d_{m+2}, \ldots$ of metrics such that $d_{m+i}$ is a metric on $Z_{m+i}$ and each $d_{m+i}$ is an extension of $d_{m}$. Moreover, we can take metrics $d_{m+i}$ on $Z_{m+i}$ satisfying the following condition: $Z_{m+i}$ is contained in the $i^{-1}$-neighborhood of $Z_{m}\left(\subset Z_{m+i}\right)$, that is, $\lim _{i \rightarrow \infty} d_{m+i}\left(Z_{m+i}, Z_{m}\right)=0$ and for any $\epsilon>0$, there is $\delta>0$ such that there is some $i_{0}$ such that if $i \geq i_{0}$ and $x \in Z_{m} \subset Z_{m+i}$, then

$$
f_{m+i}\left(U_{m+i}(x, \delta)\right) \subset U_{m+i}\left(f_{m+i}(x), \epsilon\right),
$$

where $U_{m}(x, \delta)$ (respectively $U_{m+i}(x, \delta)$ ) denotes the $\delta$-neighborhood of $x$ in $Z_{m}$ (respectively $Z_{m+i}$ ). Intuitively, we may consider that the sets $Z_{m+i}$ and maps $f_{m+i}$ converge to the set $Z_{m}\left(\subset Z_{m+i}\right)$ and the map $f_{m}: Z_{m} \rightarrow Z_{m}$, respectively. 
Set $Z_{\omega+m}=\bigoplus_{i=1}^{\infty} Z_{m+i} \oplus Z_{m}$. Define a function $f_{\omega+m}: Z_{\omega+m} \rightarrow Z_{\omega+m}$ by $f_{\omega+m}\left|Z_{m+i}=f_{m+i}, f_{\omega+m}\right| Z_{m}=f_{m}$. By using the metric $d_{m+i}$ on $Z_{m+i}$ as above $\left(i \geq 1\right.$ ), we can define a metric $d_{\omega+m}$ on $Z_{\omega+m}$ so that $d_{\omega+m}$ is an extension of $d_{m+i}$ for each $i$,

$$
\lim _{i \rightarrow \infty}\left(d_{\omega+m}\right)_{H}\left(Z_{m+i}, Z_{m}\right)=0,
$$

and the following condition is satisfied:

(†) for any $x \in Z_{m}$ and any $\epsilon>0$ there is $\delta>0$ such that

$$
f_{\omega+m}\left(U_{\omega+m}(x, \delta)\right) \subset U_{\omega+m}\left(f_{\omega+m}(x), \epsilon\right) .
$$

In particular, $f_{\omega+m}$ is continuous (see Figure 3). Note that $\Omega_{\omega}\left(f_{\omega+m}\right)=Z_{m}$. Hence $d\left(f_{\omega+m}\right)=\omega+m$.

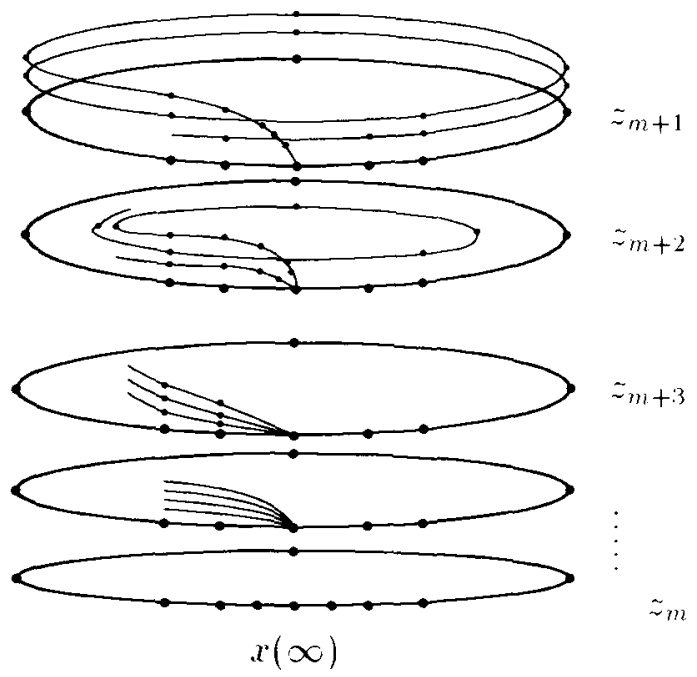

FIGURE 3

$\mathbf{I}(\alpha+m)$ : Case that $\lambda>\omega$ is a countable ordinal number which is not limit. Then we can choose the limit ordinal number $\alpha<\lambda$ such that $\lambda=\alpha+m$, where $1 \leq m<\omega$. Take a sequence $\alpha_{1}<\alpha_{2}<\alpha_{3}<\ldots$, of ordinal numbers such that $\lim _{i \rightarrow \infty} \alpha_{i}=\alpha$. Note that $\lim _{i \rightarrow \infty}\left(\alpha_{i}+m\right)=\alpha$. In this case, by induction we may assume that $Z_{\alpha_{i}+m}$ can be presented by the form $Z_{\alpha_{i}+m}=\bigoplus_{j=1}^{\infty} Z_{\beta_{i, j}+m} \oplus Z_{m}$ and the metric $d_{\alpha_{i}+m}$ satisfies $\lim _{j \rightarrow \infty}\left(d_{\alpha_{i}+m}\right)_{H}\left(Z_{\beta_{i, j}+m}, Z_{m}\right)=0$, where $\lim _{j \rightarrow \infty} \beta_{i, j}=\alpha_{i}$ (see the case $\mathrm{I}(\omega+m))$. Set

$$
Z_{\lambda}=\bigoplus_{i=1}^{\infty} Z_{\alpha_{i}+m} \oplus Z_{m}
$$


Define a function $f_{\lambda}: Z_{\lambda} \rightarrow Z_{\lambda}$ by $f_{\lambda}\left|Z_{\alpha_{i}+m}=f_{\alpha_{i}+m}, f_{\lambda}\right| Z_{m}=f_{m}$. In this case we may assume that the metrics $d_{\alpha_{i}+m},(i=1,2, \ldots)$ on $Z_{\alpha_{i}+m}$ satisfy the condition ( $\left.\dagger\right)$. By using these metrics, we can define a metric $d_{\lambda}$ on $Z_{\lambda}$ such that $d_{\lambda}$ is an extension of the metric $d_{\alpha_{i}+m}$ on $Z_{\alpha_{i}+m}, Z_{\dot{\lambda}}$ is a compactum and moreover $d_{\lambda}$ satisfies the condition $(\dagger)$. In particular, $f_{\lambda}$ is continuous (see the case $\mathrm{I}(\omega+m)$ ). Note that $\Omega_{\alpha}\left(f_{\alpha+m}\right)=Z_{m}$. Hence $d\left(f_{\alpha+m}\right)=\alpha+m$.

Next, we consider the case that $\lambda$ is a limit ordinal number. In this case, take a sequence $\alpha_{1}<\alpha_{2}<\alpha_{3}<\ldots$, of ordinal numbers such that $\alpha_{i}$ is not limit for each $i$, and $\lim _{i \rightarrow \infty} \alpha_{i}=\lambda$. For each $\alpha_{i}$ we assume that $Z_{\alpha_{i}}$ and $f_{\alpha_{i}}$ have been obtained. Set

$$
Z_{\lambda}=\bigoplus_{i=1}^{\infty} Z_{\alpha_{i}} \oplus\{\infty\} \text {. }
$$

We can define a metric $d_{i}$ on $Z_{\lambda}$ such that $\lim _{i \rightarrow \infty}\left(d_{\lambda}\right)_{H}\left(Z_{\alpha_{i}},\{\infty\}\right)=0$ and each $Z_{\alpha_{i}}\left(\subset Z_{\lambda}\right)$ is homeomorphic to $Z_{\alpha_{i}}$. Define a function $f_{\lambda}: Z_{\lambda} \rightarrow Z_{\lambda}$ by $f_{\lambda} \mid Z_{\alpha_{i}}=f_{\alpha_{i}}$, and $f_{\lambda}(\infty)=\infty$. Clearly $f_{i}$ is continuous. Then we see that $d\left(f_{\lambda}\right)=\lambda$.

Therefore, for any countable ordinal number $\lambda$ we obtained a compact countable set $Z_{\lambda}$ and a homeomorphism $f_{\lambda}: Z_{\lambda} \rightarrow Z_{\lambda}$ such that $d\left(f_{\lambda}\right)=\lambda$. This completes the proof.

LeMMA 2.2. Suppose that $A$ is a closed subset of a space $X$. Let $g: A \rightarrow A$ be a map of $A$ and $r: X \rightarrow A$ a retraction, that is, $r \mid A=\mathrm{id}$. if $f=g \cdot r: X \rightarrow X$, then $\Omega(f)=\Omega(g)$.

PROOF. Since $f \mid A=g, \Omega(g) \subset \Omega(f)$. Let $x \in \Omega(f)$. Note that $x \in A$. Suppose, on the contrary, that $x \notin \Omega(g)$. There is a neighborhood $U$ of $x$ in $A$ such that $g^{n}(U) \cap U=\phi$ for all $n \geq 1$. Since $r(x)=x$, we choose a neighborhood $V$ of $x$ in $X$ such that $r(V) \subset U$. Then

$$
\begin{aligned}
f^{n}(V) \cap V & =f^{n-1}(g \cdot r(V)) \cap V \subset f^{n-1}(g(U)) \cap V \\
& =g^{n}(U) \cap V \\
& =g^{n}(U) \cap(A \cap V) \subset g^{n}(U) \cap U=\phi .
\end{aligned}
$$

Therefore $x \notin \Omega(f)$. Hence $\Omega(f)=\Omega(g)$. If $d(g)>0$, then we see that $d(f)=$ $d(g)$.

COROLLARY 2.3. Let $C$ be a Cantor set. If $\lambda$ is any countable ordinal number, there is a map $f: C \rightarrow C$ such that $d(f)=\lambda$.

PROOF. We may assume $\lambda>0$. Let $Z_{\lambda}$ and $f_{\lambda}: Z_{\lambda} \rightarrow Z_{\lambda}$ be as in (2.1). We may assume that $Z_{\lambda} \subset C$. Then there is a retraction $r: C \rightarrow Z_{\lambda}$. Put $f=f_{\lambda} \cdot r: C \rightarrow C$. By $(2.2), \Omega\left(f_{\lambda}\right)=\Omega(f)$. Clearly $d(f)=\lambda$. 
COROLLARY 2.4. For any countable ordinal number $\lambda$ there is a 1-dimensional compactum $Y$ and a flow $\phi: Y \times R \rightarrow Y$ such that $d(\phi)=\lambda$.

PROOF. Let $Z_{\lambda}, f_{\lambda}$ be as in (2.1). Let $Y=T\left(f_{\lambda}\right)$ be the mapping torus, that is, the space obtained from $Z_{\lambda} \times I$ by identifying the points $(y, 0)$ and $\left(f_{\lambda}(y), 1\right)$. Naturally we obtain the flow $\phi$ on $Y$ from $f_{\lambda}$. Then $d(\phi)=\lambda$.

\section{The depth of centres of maps of dendrites}

In this section, we study the depth of centres of maps of some continua. The following is the main theorem of this note.

THEOREM 3.1. For any countable ordinal number $\lambda$, there is a dendrite $D$ and a map $f: D \rightarrow D$ such that the set $E(D)$ of endpoints of $D$ is a compact countable set and $d(f)=\lambda$.

PrOOF. Firstly, we define some kinds of dendrites by the following general method (see [2]): Let $X$ be a 0 -dimensional compact metric space and let $g: X \rightarrow X$ be any map of $X$. Choose an inverse sequence $X=\left\{X_{n}, p_{n \cdot n+1} \mid n=1,2, \ldots,\right\}$ of finite sets $X_{n}$ such that $X_{1}=\{*\}$ is a one point set, $p_{n, n+1}: X_{n+1} \rightarrow X_{n}$ is an onto bonding map $(n \geq 1)$ and $X=\operatorname{invlim} X$. For $1 \leq m<n$, let $p_{m, n}=p_{m, m+1} \cdots p_{n-1, n}$ and let $p_{n}: X \rightarrow X_{n}$ be the natural projection. Now, consider the infinite telescope $T(\boldsymbol{X})=\cup_{n=1}^{\infty} M\left(p_{n, n+1}\right)$, where $M\left(p_{n, n+1}\right)$ denotes the mapping cylinder of $p_{n, n+1}$ : $X_{n+1} \rightarrow X_{n}$, that is, in a topological sum $X_{n} \cup\left(X_{n+1} \times[1 /(n+1), 1 / n]\right), M\left(p_{n, n+1}\right)$ is obtained by identifying points $(x, 1 / n) \in X_{n+1} \times\{1 / n\}$ and $p_{n, n+1}(x) \in X_{n}$ for $x \in X_{n+1}$ and $T(X)$ is obtained by identifying each point of $X_{n} \times\{1 / n\}$ in $M\left(p_{n-1 . n}\right)$ and the corresponding point of $X_{n}$ in $M\left(p_{n, n+1}\right)$. Put $Y(X)=X \cup T(X)$. Define a function $\mu: Y(\boldsymbol{X}) \rightarrow I=[0,1]$ by $\mu([x, t])=t$ if $[x, t] \in T(\boldsymbol{X})$ and $\mu(x)=0$ if $x \in X$. Also, define a retraction $\psi_{t}: Y(X) \rightarrow \mu^{-1}([t, 1])(t \in I)$ by $\psi_{t}(y)=$ $\left[p_{q(t)}(x), t\right]$ for $y=x \in X, \psi(y)=\left[p_{q(t), n}(x), t\right]$ for $y=[x, s] \in \mu^{-1}((0, t])$ and $x \in X_{n}$, and $\psi_{t}(y)=y$ for $y \in \mu^{-1}([t, 1])$, where $q(t)$ is the natural number such that $1 / q(t) \leq t<1 /(q(t)-1)$. The topology of $Y(X)$ is defined by assuming that the totality of the following sets - open sets of $T(X)$ and the sets of the form $\psi_{1 / n}^{-1}(U) \cap \mu^{-1}([0,1 / n))$, where $U$ is an open set of $X_{n}(\subset Y(X)), n \geq 1-$ is an open base of $Y(\boldsymbol{X})$. Then $Y(\boldsymbol{X})$ is a compact absolute retract, and $\mu$ and $\psi_{t}$ are continuous (see [2]).

Next, for any map $g: X \rightarrow X$ we shall construct a map $f: Y(\boldsymbol{X}) \rightarrow Y(\boldsymbol{X})$ such that $f$ is an extension of $g$ and $\Omega(f)=\Omega(g) \cup\{p\}$ where $p=* \in X_{1} \subset Y(X)$. 
For each closed subset $A$ of $X$, consider the minimal subcontinuum $c(A)$ of $Y(X)$ containing $A$, that is,

$$
c(A)=\mathrm{Cl}(\cup\{[a, b]: a, b \in A\}),
$$

where $[a, b]$ is the arc from $a$ to $b$ in $Y(X)$. If an $\operatorname{arc}[a, b]$ from $a$ to $b$ is not decreasing with respect to $\mu$ (that is, if $x, y \in[a, b]$ and $a \leq x \leq y \leq b$, then $\mu(x) \leq \mu(y)$ ), we call $[a, b]$ an order arc from $a$ to $b$.

Let $\kappa(A)$ be the unique point of $c(A)$ such that $\mu(\kappa(A))=\min \{\mu(y) ; y \in c(A)\}$. Define a map $g_{1}: \cup_{n=1}^{\infty} X_{n} \rightarrow Y(X)$ such that $g_{1}(x)=\kappa\left(g\left(p_{n}^{-1}(x)\right)\right)$. By using this map $g_{1}$, we can naturally define a map $g_{2}: Y(\boldsymbol{X}) \rightarrow Y(\boldsymbol{X})$ such that $g_{2}$ is an extension of $g_{1}$ and $g$, and if $A=[a, b]$ is an order arc from $a$ to $b$, then $g_{2}(A)$ is also an order arc from $g_{2}(a)$ to $g_{2}(b)$. In this case, we say that $g_{2}$ is order-arc preserving. Choose a homeomorphism $h: I \rightarrow I$ such that $h(0)=0, h(1)=1$, and $h(t)>t$ for $0<t<1$; for example, $h(t)=\sqrt{t}$. Define a function $f: Y(X) \rightarrow Y(X)$ by

$$
f(y)=\psi_{h \cdot \mu(y)}\left(g_{2}(y)\right) .
$$

Then $f$ is continuous and $f(p)=p, f \mid X=g$ and $f$ is order-arc preserving. Also, note that if $y \in Y(X)-(X \cup\{p\})$, then $\mu(y)<\mu(f(y))$.

Next, we show that $\Omega(f)=\Omega(g) \cup\{p\}$. Let $x \in \Omega(f)$. Since $y<\mu(y)$ for any $y \in Y(X)-(X \cup\{p\})$, we see that $x \in X \cup\{p\}$. Suppose, on the contrary, that $x \notin \Omega(g) \cup\{p\}$. Then there is a neighborhood $U$ of $x$ in $X$ such that $g^{n}(U) \cap U=\phi$ for all $n \geq 1$. Take a point $x_{n} \in X_{n}$ such that $x \in \psi_{1 / n}^{-1}\left(x_{n}\right) \cap X \subset U$. Set $V=\psi_{1 / n}^{-1}\left(x_{n}\right)$. Then $f^{n}(V) \cap V=\phi$ for all $n \geq 1$. In fact, suppose, on the contrary, that there is $y \in V$ such that $f^{n}(y) \in V$ for some $n \geq 1$. Choose a point $y^{\prime} \in X \cap V$ such that $\left[y^{\prime}, y\right]$ is an order arc. Then $\left[f^{n}\left(y^{\prime}\right), f^{n}(y)\right]$ is an order arc. Since $f^{n}(y) \in V$, $f^{n}\left(y^{\prime}\right) \in V \cap X \subset U$, which implies that $g^{n}(U) \cap U \neq \phi$. This is a contradiction. Hence $x \in \Omega(g) \cup\{p\}$.

Suppose that $\lambda>0$ is any countable ordinal number. Choose a compact countable set $X=Z_{\lambda}$ and a homeomorphism $g=f_{\lambda}: X=Z_{\lambda} \rightarrow X=Z_{\lambda}$ such that $d\left(f_{\lambda}\right)=\lambda$. In this case, we may assume that for each $n \geq 2\left|X_{n}\right| \geq 2$, where $\left|X_{n}\right|$ denotes the cardinality of $X_{n}$. Then we obtain a map $f: D=Y(X) \rightarrow D$ such that $\Omega(f)=\Omega\left(f_{\lambda}\right) \cup\{p\}$ and $E(D)=Z_{\lambda}$. Hence $d(f)=\lambda$.

By (2.2), we obtain the following.

COROLLARY 3.2. There is a dendrite $D$ such that for any countable ordinal number $\lambda$, there is a map $f: D \rightarrow D$ such that $d(f)=\lambda$.

COROLLARY 3.3. For any countable ordinal number $\lambda$, there is a map $f: B^{2} \rightarrow B^{2}$ of a disk (=2-dimensional ball) $B^{2}$ such that $d(f)=\lambda$. 
PROOF. Let $\lambda>0$ be any countable ordinal number. By (3.1), we can choose a map $g: D \rightarrow D$ of a dendrite $D$ such that $d(g)=\lambda$. Since $D$ is a dendrite, we may assume that $D \subset B^{2}$. Since $D$ is an $\mathrm{AR}$, there is a retraction $r: B^{2} \rightarrow D$. Put $f=g \cdot r: B^{2} \rightarrow B^{2}$. Then $d(f)=\lambda$.

In [5], Neumann proved that for any $C^{\infty} n$-manifold $M$ with $n \geq 3$ and any countable ordinal number $\lambda$, there is a $C^{\infty}$ flow $\phi$ on $M$ such that the depth of the centre of $\phi$ is $\lambda$.

Here, we prove the following.

COROLLARY 3.4. For any countable ordinal number $\lambda$, there is a homeomorphism $h: B^{3} \rightarrow B^{3}$ of a 3-dimensional ball $B^{3}$ such that $h \mid \partial B^{3}=\mathrm{id}, d(h)=\lambda$ and $\Omega_{\lambda}(h)=\partial B^{3} \cup Z$, where $Z$ is a countable compactum in $B^{3}-\partial B^{3}$.

PROOF. We may assume that $B^{3}=B^{2} \times[-1,1]$. Choose a compact countable set $Z_{\lambda}$ and a homeomorphism $f_{\lambda}: Z_{\lambda} \rightarrow Z_{\lambda}$ such that $d\left(f_{\lambda}\right)=\lambda$. We may assume that $X=Z_{\lambda} \subset\left(B^{2}-\partial B^{2}\right) \times\{0\}$. By [3, Chapter 13], we can choose a homeomorphism $g: B^{2} \rightarrow B^{2}$ such that $g$ is an extension of $f$ and $g \mid \partial B=$ id. We can choose a map $\psi: B^{2} \times[-1,1] \rightarrow[-1,1]$ satisfying the following conditions:

(1) $\psi(x, t)=t$ for $x \in \partial B^{2} \times[-1,1]$,

(2) $\psi(x,-1)=-1, \psi(x, 1)=1$ for each $x \in B^{2}$,

(3) $\psi(x, t)>t$ if $x \notin X,-1<t<1$,

(4) $\psi(x, 0)=0$ if $x \in X$, and

(5) $\psi(x, t)>t$ if $x \in X, t \neq-1,0,1$.

Consider the suspension $S\left(B^{2}\right)$ of $B^{2}$, that is, $S\left(B^{2}\right)$ is the quotient space of $B^{2} \times[-1,1]$ in which $B \times\{-1\}$ and $B^{2} \times\{1\}$ are identified to two different points. If $(x, t) \in B^{2} \times[-1,1]$, we use $[x, t]$ to denote the corresponding point of $S\left(B^{2}\right)$ under the quotient map $q: B^{2} \times[-1,1] \rightarrow S\left(B^{2}\right)$. Note that $S\left(B^{2}\right)=B^{3}$ is a 3-dimensional ball. Define a homeomorphism $h: B^{3} \rightarrow B^{3}$ by $h([x, t])=[g(x), \psi(x, t)]$.

Suppose that $(x, t) \in\left(B^{2} \times[-1,1]\right)-\left(X \cup \partial\left(B^{2} \times[-1,1]\right)\right)$. Since $\psi(x, t)>t$, we can choose a neighborhood $U$ of $(x, t)$ such that $\psi(U) \cap p(U)=\phi$, where $p: B^{2} \times[-1,1] \rightarrow[-1,1]$ is the natural projection. Since $\psi(x, t)$ is not decreasing with respect to $t$, we see that $h^{n}(q(U)) \cap q(U)=\phi$ for all $n \geq 1$. Hence $\Omega(h) \subset$ $X \cup \partial B^{3}$. If $\lambda$ is any ordinal number with $\lambda \geq \omega$, then we see that $d(h)=\lambda$. Suppose that $0<\lambda=m<\omega$. In this case, moreover, we can choose a homeomorphism $g: B^{2} \rightarrow B^{2}$ such that there is a small disk $D^{\prime}$ which is a neighborhood of $x_{m}(0) \in$ $Z_{m}-Z_{m-1}$ satisfying $g^{i}\left(D^{\prime}\right) \cap g^{i}\left(D^{\prime}\right)=\phi(i \neq j), g^{i}\left(D^{\prime}\right) \cap Z_{m-1}=\phi$ for each $i$, and $\lim _{n \rightarrow \pm \infty} \operatorname{diam}\left(g^{i}\left(D^{\prime}\right)\right)=0$ (see Figure 4 and the proof of [3, Theorem 1, p. 91]), where $Z_{0}=\{x(\infty)\}$. Then $\Omega(h)=\partial\left(B^{3}\right) \cup \Omega\left(f_{\lambda}\right)=\partial B^{3} \cup Z_{m-1}$. Hence we see that $d(h)=m$. 


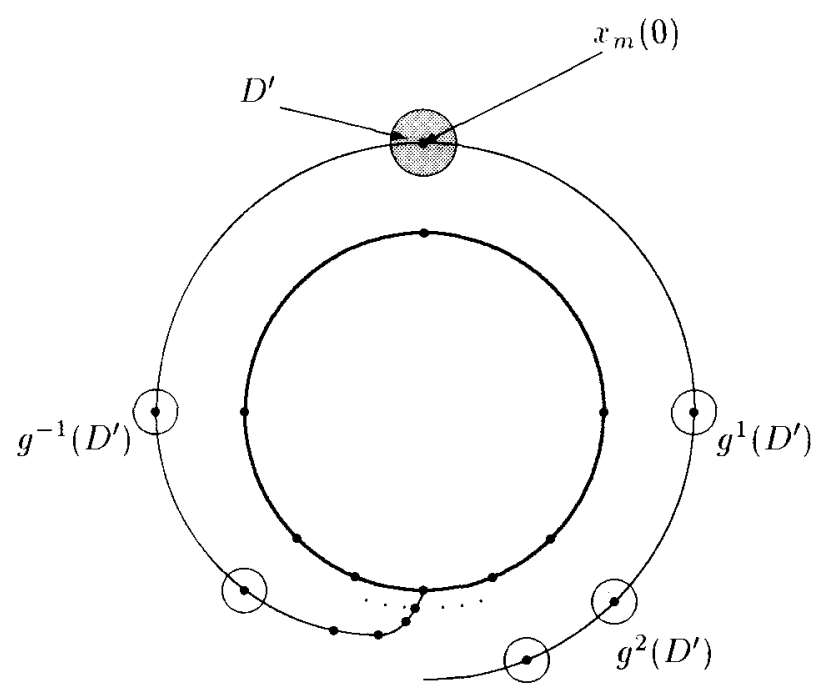

FIGURE 4

\section{References}

[1] H. Kato, 'A note on periodic points and recurrent points of maps of dendrites', Bull. Austral. Math. Soc. 51 (1995), 459-461.

[2] J. Krasinkiewicz, 'On a method of constructing ANR-sets. An application of inverse limits', Fund. Math. 92 (1976), 95-112.

[3] E. E. Moise, Geometric topology in dimension 2 and 3, Graduate Texts in Math. 47 (Springer, Berlin, 1977).

[4] S. B. Nadler, Jr., Continuum theory, Pure Appl. Math. 158 (Wiley, New York, 1992).

[5] D. A. Neumann, 'Central sequences in dynamical systems', Amer. J. Math. 100 (1978), 1-18.

[6] J. C. Xiong, ' $\Omega(f \mid \Omega(f))=\overline{P(f)}$ for every continuous self-map $f$ of the interval', Kexue Tongbao 28 (1983), 21-23.

[7] X. D. Ye, 'The center and the depth of the center of a tree map', Bull. Austral. Math. Soc. 48 (1993), 347-350.

Institute of Mathematics

University of Tsukuba

Ibaraki 305

Japan

e-mail: hisakato@sakura.cc.tsukuba.ac.jp 